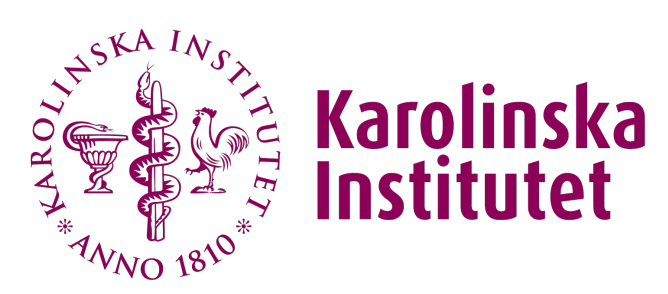

Karolinska Institutet

http://openarchive.ki.se

This is a Peer Reviewed Accepted version of the following article, accepted for publication in International Journal of Cancer.

\title{
A possible link between famine exposure in early life and future risk of gastrointestinal cancers : implications from age-period-cohort analysis
}

Xie, Shao-Hua; Lagergren, Jesper

Int J Cancer. 2016 Oct 25.

http://doi.org/10.1002/ijc.30485

http://hdl.handle.net/10616/45373

If not otherwise stated by the Publisher's Terms and conditions, the manuscript is deposited under the terms of the Creative Commons Attribution-NonCommercial-NoDerivatives License (http://creativecommons.org/licenses/by-nc-nd/4.0/), which permits non-commercial re-use, distribution, and reproduction in any medium, provided the original work is properly cited, and is not altered, transformed, or built upon in any way. 


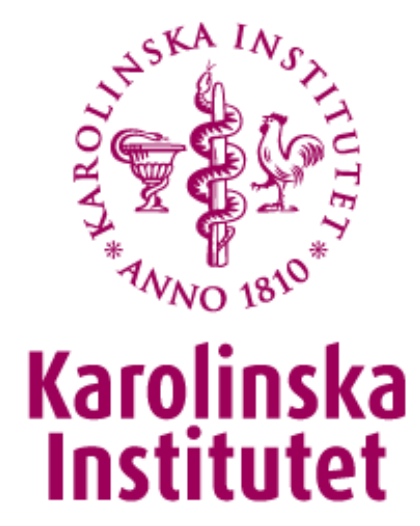

This is an author produced version of a paper accepted by International Journal of Cancer. This paper has been peerreviewed but does not include the final publisher proofcorrections or journal pagination.
A possible link between famine exposure in early life and future risk of gastrointestinal cancers : implications from age-period-cohort analysis
Shao-Hua, Xie; Jesper, Lagergren
Access to the published version may require subscription.
Published with permission from: Wiley 


\section{A Possible Link between Famine Exposure in Early Life and Future Risk of Gastrointestinal Cancers: Implications from Age-Period-Cohort Analysis}

Short title: Famine and GI cancers

Authors: Shao-Hua Xie ${ }^{1}$, and Jesper Lagergren ${ }^{1,2}$

Affiliations: ${ }^{1}$ Upper Gastrointestinal Surgery, Department of Molecular Medicine and Surgery, Karolinska Institutet, Karolinska University Hospital, Stockholm, Sweden; and ${ }^{2}$ Division of Cancer Studies, King's College London, London, United Kingdom

Correspondence to: Dr. Shao-Hua Xie, Upper Gastrointestinal Surgery, Department of Molecular Medicine and Surgery, Karolinska Institutet, NS 67, $2^{\text {nd }}$ Floor, Stockholm 17176, Sweden; Tel: +46 8517 70917; Fax: +46 8517 76280; Email: shaohua.xie@ki.se

Key words: famine; gastrointestinal cancer; Chinese Famine; starvation; nutrition deficiency

Abbreviations: ASR, age-standardized incidence rate; CI, confidence interval.

\section{Novelty and Impact}

The Chinese famine in 1958-1962 was one of the worst in history, but its influence on cancer risks is uncertain. This age-period-cohort analysis based on cancer incidence data in Shanghai suggests exposure to famine in early life increases the risk of multiple gastrointestinal cancers. The particularly increased risk of esophageal, liver, and colorectal cancer indicates a vulnerable exposure period in early childhood. The findings need to be confirmed in further investigations at an individual level. 


\begin{abstract}
The Chinese famine in 1958-1962 was one of the worst in human history, but its potential influence on cancer risks is uncertain. Using cancer incidence data in Shanghai, China, during 1983-2007, we calculated age-specific incidence rates of gastrointestinal cancers in birth cohorts exposed to the Chinese famine in different periods of life and a non-exposed reference cohort. Age-period-cohort regressions estimated the overall relative risks of gastrointestinal cancers in each birth cohort. A total of 212,098 new cases of gastrointestinal cancer were identified during the study period (129,233 males and 82,865 females), among whom 18,146 had esophageal cancer, 71,011 gastric cancer, 55,864 colorectal cancer, 42,751 liver cancer, 9,382 gallbladder cancer, and 14,944 had pancreatic cancer. The risk of esophageal, gastric, colorectal, and liver cancers was higher in cohorts exposed to the Chinese famine in early life than in the reference cohort, except for esophageal cancer in women. The risk of esophageal, liver, and colorectal cancers was particularly high in men exposed to famine during early childhood (0-9 years). There were no clear associations between famine exposure and the risk of pancreatic or gallbladder cancer. This study suggests an increased risk of esophageal, gastric, liver, and colorectal cancers associated with childhood exposure to the Chinese famine. These findings indicate a need for further investigations confirming the results and identifying the underlying mechanisms.
\end{abstract}


The fetal origin hypothesis, also known as "Barker's hypothesis" or "thrifty phenotype hypothesis", postulates that fetal undernutrition leads to restricted fetal growth metabolic adaptations, which may result in increased risk of chronic diseases later in life ${ }^{1,2}$. Famines can be seen as natural experiments that can provide unique opportunities to investigate the long-term health effects of nutritional deprivation during fetal life and early childhood. Previous famine studies have examined associations between famine exposure in early life and risks of a variety of common diseases during adulthood, including hypertension ${ }^{3}$, obesity ${ }^{4}$, type 2 diabetes and other metabolic disorders ${ }^{5-7}$, and respiratory diseases ${ }^{8}$. However, only a limited number of studies, mostly from Europe, have addressed the risk of cancer in adulthood associated with famine exposure in early life, and these provided inconsistent results ${ }^{9-15}$.

The Chinese famine, lasting from the late 1950s to the early 1960s was one of the worst famines in human history, affecting the entire Chinese nation and causing millions of deaths ${ }^{16}$. Recent studies have linked exposure to the Chinese famine in early life with increased risks of overweight ${ }^{17}$, hypertension ${ }^{18}$, metabolic disorders ${ }^{19,20}$, and schizophrenia ${ }^{21}$, whereas evidence regarding cancer risks is limited. Gastrointestinal cancers represent a group of common malignancies globally and they are the leading causes of cancer-related deaths in China. Approximately half of all cases of esophageal, gastric, and liver cancers occur in China ${ }^{22}$. The past few decades have also witnessed a continuous rise in the incidence of colorectal, gallbladder, and pancreatic cancers in China ${ }^{23}$. We aimed to examine the associations between famine exposure in early life and the risk of gastrointestinal cancers in adulthood in a Chinese population, using cancer incidence data in Shanghai, China. 


\section{Materials and Methods}

\section{$\underline{\text { Data sources }}$}

We extracted data on the incidence of six types of gastrointestinal cancer and population sizes in Shanghai, China, during the period 1983-2007 from the Cancer Incidence in Five Continents (CI5) series volumes VI to X which are published by the International Agency for Research on Cancer (IARC) ${ }^{24-28}$. Starting in 1963, the Shanghai Cancer Registry is the earliest population-based cancer registry in China and cancer incidence data from this registry have been included in the CI5 series since 1975 . The cancer types were defined by diagnosis codes from the seventh version of the International Classification of Diseases: cancer of the esophagus (code 150), stomach (151), liver (155.0), gallbladder (155.1), pancreas (157), and colon and rectum (153-154).

\section{$\underline{\text { Statistical analysis }}$}

We computed the crude incidence rates and age-standardized rates by sex and anatomical site for each CI5 volume in five-year calendar periods. The age-standardized rates were calculated using the direct method with the World Health Organization (WHO) World Standard Population 2000 as reference ${ }^{29}$. The $95 \%$ confidence intervals (CIs) of crude rates were estimated assuming a Poisson distribution, while CIs for age-standardized rates were calculated based on the gamma distribution, as it assumes that the standardized rate is a weighted sum of independent Poisson random variables ${ }^{30}$. We used age-period-cohort models to differentiate the effects of age, calendar period, and birth cohort on incidence rates of gastrointestinal cancers ${ }^{31}$. Since incidence data were available in 5-year age groups, we used the equivalent 5-year calendar periods, and consequently, spanned into partially overlapping 9-year birth cohorts. For example, those who were diagnosed with cancer at the ages of 40-44 and 45-49 years during the period 2003-2007 were categorized into the birth cohorts 1959- 
1967 and 1964-1972, respectively. We defined the following birth cohorts exposed to the Chinese famine (1958-1962) in different periods of life: 1954-1962 (exposed at ages 0-9 years), 1949-1957 (exposed at ages 1-13 years), 1944-1952 (exposed at ages 6-18 years), and 1939-1947 (exposed at ages 11-23 years), in addition to a reference birth cohort without famine exposure (born during 1964-1972). A Lexis diagram presents the data matrix (Supplementary Figure 1). The age-specific incidence rates of gastrointestinal cancers were calculated by sex and site for each birth cohort. We further performed age-period-cohort regressions on the incidence of gastrointestinal cancers by sex and site using a web tool, newly developed by the National Cancer Institute (NCI), United States ${ }^{32}$. The age-periodcohort regression is based on a log-linear model for the incidence rates with additive effects from age, calendar period, and birth cohort as shown in the formula below:

$$
\rho_{p a}=\alpha_{a}+\beta_{p}+\gamma_{c}
$$

where $\rho_{p a}$ is the expected incidence rate, and $\alpha_{a}, \beta_{p}$, and $\gamma_{c}$ indicate the effects of age, period, and cohort, respectively.

The generic additive effects can be partitioned into linear and non-linear components. The used web tool fits the age-period-cohort model, and estimates the overall annual percentage changes (net drifts) in age-standardized rates which incorporate linear components of both cohort and period effects ${ }^{31}$. We plotted the relative risks of gastrointestinal cancers and the corresponding 95\% CIs for each birth cohort by sex and site, adjusted for age and non-linear period effects. The relative risk estimates for the central categories of consecutive age groups, calendar periods, and birth cohorts were constrained to 1 (reference value) in all age-periodcohort analyses, respectively. In the case of an even number of age groups, calendar periods, or birth cohorts, we used the lower of the two central categories as the reference. We performed all statistical analyses using the statistical software SAS version 9.4 (SAS Institute, Cary, NC), except for age-period-cohort analyses. 


\section{Results}

A total of 212,098 new cases of gastrointestinal cancers $(129,233$ [61\%] males and 82,865 [39\%] females) occurred during the period 1983-2007 in Shanghai. These included 18,146 (9\%) cases of esophageal cancer, 71,011 (33\%) of gastric cancer, 55,864 (26\%) of colorectal cancer, 42,751 (20\%) of liver cancer, 9,382 (4\%) of gallbladder cancer, and 14,944 (7\%) cases of pancreatic cancer (Table 1).

\section{Esophageal cancer}

The age-standardized rates of esophageal cancer declined from 17.3 to 7.8 per 100,000 person-years in males and from 7.4 to 2.4 per 100,000 person-years in females in 1983-2007, resulting in an average annual decrease of $3.4 \%$ and $6.8 \%$, respectively (Table 1). The agespecific incidence rates of esophageal cancer in men were generally higher in the birth cohort with famine exposure at ages 0-9 years (born during 1954-1962) than in birth cohorts exposed to the famine later in life, as well as in the reference cohort without famine exposure (Figure 1). The overall risk of esophageal cancer in men increased with each successive birth cohort from 1939-1947 until peaking with the cohort with famine exposure at 0-9 years of age, after which it declined (Figure 2). The rates in women were relatively low in all birth cohorts and decreased, in general, with successive birth cohorts (Figures 1 and 2).

\section{Gastric cancer}

The age-standardized rates of gastric cancer decreased on average $3.4 \%$ and $6.8 \%$ per year in males and females, respectively, during the study period (Table 1). The age-specific incidence rates were higher in birth cohorts with famine exposure than in the reference cohort in both sexes, and the rates were similar among the cohorts with famine exposure in different periods of life (Figure 1). The overall risk of gastric cancer generally decreased with successive birth 
cohorts in both sexes, but did not decrease in the four cohorts (1939-1947 to 1954-1962) exposed to famine in early life (Figure 2).

\section{$\underline{\text { Colorectal cancer }}$}

The age-standardized rates of colorectal cancer increased from 20.1 to 32.2 per 100,000 person-years in males and from 17.5 to 26.7 per 100,000 person-years in females, resulting in an average annual increase of $0.3 \%$ in both sexes (Table 1). The age-specific incidence rates were higher in birth cohorts exposed to famine compared to the unexposed reference cohort in both sexes (Figure 1). The age-specific rates were similar among the cohorts exposed to famine despite seemingly increased rates at ages $45-49$ years and above in cohorts exposed in earlier periods of life in men (Figure 1). The overall risk of colorectal cancer increased with each successive exposed birth cohort, and declined from the cohort born during 1959-1967 (Figure 2).

\section{$\underline{\text { Liver cancer }}$}

The age-standardized rates of liver cancer decreased from 33.8 to 24.3 per 100,000 personyears in males and from 12.1 to 8.2 per 100,000 person-years in females, thus resulting in an average annual decrease of $1.8 \%$ and $2.3 \%$, respectively (Table 1 ). The age-specific incidence rates were higher in birth cohorts exposed to famine in early life compared to those in the unexposed cohort in both sexes. The rates in men were particularly increased in the birth cohort exposed to famine at ages 0-9 years (born during 1954-1962) (Figure 3). The overall risk of liver cancer in men increased with successive birth cohorts from 1944-1952 and peaked with the cohort exposed to famine at ages 0-9 years, and declined thereafter. In women, the risk decreased with successive birth cohorts, except for similar risks in three cohorts with famine exposure in early life (born during 1944-1952 to 1954-1962) (Figure 4). 


\section{Gallbladder cancer}

The age-standardized rates of gallbladder cancer increased from 2.3 to 5.9 per 100,000 person-years in males and from 3.5 to 5.1 per 100,000 person-years in females, and the average annual increase was $0.9 \%$ and $0.4 \%$, respectively (Table 1). The age-specific incidence rates were similar across birth cohorts in men, while the rates in women were slightly higher in the birth cohort with famine exposure at ages 0-9 years (born during 19541962) compared to the other birth cohorts (Figure 3). We observed no significant birth cohort effects in the cohorts born during1919-1927 or later in men. The overall risk of gallbladder cancer was higher in the birth cohort exposed to famine at ages 0-9 years (born during 19541962) compared to cohorts exposed later in life and the reference cohort, but the estimates had limited precision (Figure 4).

\section{$\underline{\text { Pancreatic cancer }}$}

The age-standardized rates of pancreatic cancer increased from 6.8 to 8.3 per 100,000 personyears in males and from 4.6 to 6.3 per 100,000 person-years in females, resulting in an average annual increase of $0.6 \%$ and $1.4 \%$, respectively (Table 1). The age-specific incidence rates were slightly higher in the birth cohort exposed to famine at ages 0-9 years (born during1954-1962) than in two cohorts exposed to famine later in life (born during 1949-1957 and 1944-1952) (Figure 3). There were no significant birth cohort effects in the cohorts born during 1919-1927 or later, except for an upward shift for the cohort born during 1959-1967 in females (Figure 4). 


\section{Discussion}

This study suggests that exposure to famine in early life increases the risk of several gastrointestinal cancers, including esophageal, gastric, liver, and colorectal cancers, compared with a birth cohort without famine exposure, despite that the increased risks of esophageal and liver cancers were mainly restricted to men. The risk of esophageal, liver, and colorectal cancers was particularly high in men exposed to famine in early childhood (0-9 years) compared to birth cohorts exposed to famine later in life.

Previous research examining famine exposure in the etiology of cancer is sparse and mainly originates from European countries. Some findings indicate an increased overall cancer risk and breast cancer risk in survivors of the Dutch Hunger Winter famine in 1944-1945 and the Siege of Leningrad in 1941-1944, Israeli Jewish survivors of World War II, and in women in Guernsey during the German occupation in 1940-1945 9,10,13-15. Only a few studies have examined potential associations between famine exposure and the risk of gastrointestinal cancers and the available results are inconsistent, possibly due to the limited number of cancer cases ${ }^{12-14}$. A decreased risk of colorectal cancer has been indicated in men exposed to the Dutch Hunger Winter ${ }^{12}$, while an increased risk has been suggested among Israeli Jewish survivors of World War II ${ }^{13}$, No altered risks of gastric or colorectal cancer were found in survivors of the Siege of Leningrad ${ }^{14}$. To the best of our knowledge, only one previous study has examined the association between exposure to the Chinese famine in early life and later cancer risk, indicating an increased mortality rate of gastric cancer ${ }^{33}$. This finding is in line with the present study, but in contrast to our findings, no critical exposure period was found in the previous study. The influence of exposure to the Chinese famine on other gastrointestinal cancer types has not been analyzed prior to the present study. The inconsistency in previous findings may be explained by differences in the severity and duration of the famine exposure, 
study design, populations, and histological tumour subtypes, as well as chance due to limited exposed cohort sizes in some studies.

The present study is the first to investigate the associations between exposure to the Chinese famine and the risk of different types of gastrointestinal cancer. The mechanisms behind the increased risk of certain gastrointestinal cancers in the birth cohorts exposed to the Chinese famine in early life in the present study are unknown, but restricted intake of foods or nutrients with protective effect against cancer risk, e.g., lower intake of fruit, vegetables and vitamins, could play an important role ${ }^{34}$. It has been speculated that mammals adjust their life history strategy for poor conditions later in life in response to an adverse environment in prenatal development, but there might be adverse consequences if there are mismatches between the anticipated and the actual mature environment ${ }^{35}$. Such predictive adaptive response and mismatch theories seem to support the observed elevated risks of gastrointestinal cancers in birth cohorts who were exposed to starvation in early life, but experienced great socioeconomic improvements later in life due to the rapid socioeconomic developments in recent decades in China. ${ }^{36,37}$ Other potential explanations for our findings include physical injury to the digestive tract caused by ingesting very coarse food (for esophageal cancer), increased occurrence of peptic ulcer (for gastric cancer), increased prevalence of hepatitis (for liver cancer), and intake of food contaminated with aflatoxins (for liver cancer) during the famine period. These hypotheses remain to be examined. Furthermore, previous research has shown that those who had exposure to the Chinese famine in early life are more likely to have lower income, less education, and short labour supply, which may cause psychological stress ${ }^{38}$. It has been shown that Chinese famine exposure increases the risk of obesity ${ }^{17,39}$, diabetes ${ }^{20}$, and hypertension ${ }^{18}$. All these conditions are associated with an increased cancer risk ${ }^{40,41}$, and may have contributed to the observed association between famine exposure and cancer 
risk in this study. Moreover, China has experienced dramatic demographic and socialeconomic changes between the 1950s and 1960s, which might have influenced cancer risk in the population and remain to be identified in further studies. Changes of cancer risk in birth cohorts earlier than the 1940s are probable to be explained by other exposures rather than famine, which is not within the scope of this study and not further discussed hereby.

We observed particularly high risk of esophageal, liver, and colorectal cancers associated with famine exposure in early childhood, indicating a critical exposure period in early childhood. This observation is biologically feasible given the unique vulnerability of children to environmental exposures ${ }^{42,43}$. The reasons for the stronger excess risks of gastrointestinal cancers in men than in women are unclear. There is a male predominance in gastrointestinal cancers, particularly in esophageal, gastric, and liver cancer, for which sex hormones have been suggested to play a role ${ }^{22,44}$. It is likely that intrinsic differences between the sexes, e.g., sex hormones, mediate the associations between famine exposure and risk of gastrointestinal cancers. Interactions between famine exposure and other environmental risk factors, e.g., famine exposure enhancing the effects of alcohol drinking and tobacco smoking on the risk of esophageal cancer, are other possible explanations for the stronger associations in men than in women in the present study. Future studies exploring sex difference in the associations between early famine exposure and cancer risks in adulthood, and the underlying mechanisms, are needed to clarify these issues.

Some methodological issues deserve certain attention. The data from the cancer register used were of good quality in terms of completeness of coverage and accuracy, which lends validity to the findings. Moreover, age-period-cohort analyses may have provided an assessment of the birth cohort-specific risk with adjustment for non-linear period effects which may be 
associated with artificial changes of incidence rates ${ }^{31,32}$. A limitation is the descriptive analyses at population level without the possibility of making inferences at the individual level. Given the uncertainty in defining the year of birth based on information on the age of diagnosis and the calendar period of diagnosis, the defined birth cohorts spanned over a longer duration in 9-year intervals and partially overlap with each other. However, such random misclassification, which was independent from the cancer diagnosis, would have diluted the associations between famine exposure and cancer risks and not explained the associations identified. Moreover, the collinearity among age, period, and cohort effects in age-period-cohort analyses needs to be considered when interpreting the results ${ }^{31,32}$. An individual records approach would probably overcome the inherited limitations of age-periodcohort analysis based on surveillance data and generate more convincing evidence. Further analyses from researchers for whom such data are made available are encouraged. The Chinese famine of 1958-1962 affected the entire Chinese nation, but was more severe in rural areas 45 . There has been a substantial interprovincial migration from less-developed areas to Shanghai in recent decades. These migrants were mainly from provinces seriously starving during the Chinese famine, i.e., Anhui (29\%), Jiangsu (17\%), Henan (9\%), and Sichuan (7\%), and approximately $80 \%$ of all migrants were from rural areas ${ }^{46}$. Therefore, the observed association between famine exposure and cancer risk in this study may be explained by famine effects in these migrants, which accounted for approximately $25 \%$ of the total population ${ }^{47}$, more than in the native Shanghai residents. Our findings remain to be confirmed in other Chinese populations, preferably in rural populations that may have experienced more severe famine exposure. Providing there is more convincing evidence supporting the link between famine exposure and cancer risks, these observations need to be examined using analytic investigations at the individual level, i.e., case-control or cohort studies. Another limitation is that the analyses were restricted to a single register, which 
decreases the generalizability of the results. Yet, the register used is the only one with a history of good quality long enough to assess the Chinese famine exposure. In addition, we did not have access to data later than for the period 2003-2007, and thus, age-specific incidence rates for the reference birth cohort were only available until the age of 35-39 years. Only when more recent incidence data become available, it will be possible to compare the incidence rates across birth cohorts for older age groups of diagnosis. Finally, subgroup analyses by histological type of cancer were not possible due to lack of such information. However, the vast majority of esophageal cancer in China is squamous cell carcinoma and the remaining upper gastrointestinal cancer types studied are all strongly dominated by the adenocarcinoma subtype.

In summary, the present study suggests a possible link between exposure to the Chinese famine during childhood and an increased risk of esophageal, gastric, liver, and colorectal cancers. The particularly increased risk of esophageal, liver, and colorectal cancer after exposure to famine occurring at 0-9 years of age indicates a vulnerable exposure period in early childhood. However, these descriptive findings need to be confirmed in further investigations, and in studies that can evaluate the risk at an individual level and assess underlying mechanisms. 


\section{Acknowledgements}

This work is supported by the Swedish Research Council (SIMSAM) [D0547801] and the Swedish Cancer Society [14 0322] to JL. 


\section{References}

1. de Boo HA, Harding JE. The developmental origins of adult disease (Barker) hypothesis. Aust N Z J Obstet Gynaecol 2006;46(1):4-14.

2. Gluckman PD, Hanson MA, Pinal C. The developmental origins of adult disease. Matern Child Nutr 2005;1(3):130-41.

3. Koupil I, Shestov DB, Sparen P, et al. Blood pressure, hypertension and mortality from circulatory disease in men and women who survived the siege of Leningrad. Eur J Epidemiol 2007;22(4):223-34.

4. Ravelli AC, van Der Meulen JH, Osmond C, et al. Obesity at the age of $50 \mathrm{y}$ in men and women exposed to famine prenatally. Am J Clin Nutr 1999;70(5):811-6.

5. Lumey LH, Khalangot MD, Vaiserman AM. Association between type 2 diabetes and prenatal exposure to the Ukraine famine of 1932-33: a retrospective cohort study. Lancet Diabetes Endocrinol 2015;3(10):787-94.

6. de Rooij SR, Roseboom TJ, Painter RC. Famines in the last 100 years: implications for diabetes. Curr Diab Rep 2014;14(10):536.

7. Roseboom TJ, van der Meulen JH, Osmond C, et al. Plasma lipid profiles in adults after prenatal exposure to the Dutch famine. Am J Clin Nutr 2000;72(5):1101-6.

8. Lopuhaa CE, Roseboom TJ, Osmond C, et al. Atopy, lung function, and obstructive airways disease after prenatal exposure to famine. Thorax 2000;55(7):555-61.

9. Ekamper $P$, van Poppel $F$, Stein $A D$, et al. Prenatal famine exposure and adult mortality from cancer, cardiovascular disease, and other causes through age 63 years. Am J Epidemiol 2015;181(4):271-9.

10. Elias SG, Peeters PH, Grobbee DE, et al. Breast cancer risk after caloric restriction during the 1944-1945 Dutch famine. J Natl Cancer Inst 2004;96(7):539-46.

11. Elias SG, Peeters PH, Grobbee DE, et al. The 1944-1945 Dutch famine and subsequent overall cancer incidence. Cancer Epidemiol Biomarkers Prev 2005;14(8):1981-5.

12. Hughes LA, van den Brandt PA, Goldbohm RA, et al. Childhood and adolescent energy restriction and subsequent colorectal cancer risk: results from the Netherlands Cohort Study. Int J Epidemiol 2010;39(5):1333-44.

13. Keinan-Boker L, Vin-Raviv N, Liphshitz I, et al. Cancer incidence in Israeli Jewish survivors of World War II. J Natl Cancer Inst 2009;101(21):1489-500.

14. Koupil I, Plavinskaja S, Parfenova N, et al. Cancer mortality in women and men who survived the siege of Leningrad (1941-1944). Int J Cancer 2009;124(6):1416-21.

15. Fentiman IS, Allen DS, Ellison GT. The impact of the Occupation of Guernsey 1940-1945 on breast cancer risk factors and incidence. Int J Clin Pract 2007;61(6):937-43.

16. Smil V. China's great famine: 40 years later. BMJ 1999;319(7225):1619-21.

17. Wang $Y$, Wang $X$, Kong $Y$, et al. The Great Chinese Famine leads to shorter and overweight females in Chongqing Chinese population after 50 years. Obesity (Silver Spring) 2010;18(3):588-92. 18. Li Y, Jaddoe VW, Qi L, et al. Exposure to the Chinese famine in early life and the risk of hypertension in adulthood. J Hypertens 2011;29(6):1085-92.

19. Li Y, Jaddoe VW, Qi L, et al. Exposure to the chinese famine in early life and the risk of metabolic syndrome in adulthood. Diabetes Care 2011;34(4):1014-8.

20. Li Y, He Y, Qi L, et al. Exposure to the Chinese famine in early life and the risk of hyperglycemia and type 2 diabetes in adulthood. Diabetes 2010;59(10):2400-6.

21. Altschuler EL. Schizophrenia and the Chinese famine of 1959-1961. JAMA 2005;294(23):2968; discussion -9 .

22. GLOBOCAN 2012 v1.0, Cancer Incidence and Mortality Worldwide: IARC CancerBase No. 11 [database on the Internet]. International Agency for Research on Cancer. 2013 [cited 2015-12-23]. Available from: http://globocan.iarc.fr, accessed on 12/23/2015.

23. Zhao P, Dai M, Chen W, et al. Cancer trends in China. Jpn J Clin Oncol 2010;40(4):281-5. 
24. Curado MP, Edwards B, Shin HR, et al. Cancer Incidence in Five Continents, Vol. IX, IARC Scientific Publications No. 160. Lyon: International Agency for Research on Cancer; 2007.

25. Parkin DM, Muir CS, Whelan SL, et al. Cancer Incidence in Five Continents, Vol. VI, IARC Scientific Publications No. 120. Lyon: International Agency for Research on Cancer; 1992.

26. Parkin DM, Whelan SL, Ferlay J, et al. Cancer Incidence in Five Continents, Vol. VII, IARC Scientific Publications No. 143. Lyon: International Agency for Research on Cancer; 1997.

27. Parkin DM, Whelan SL, Ferlay J, et al. Cancer Incidence in Five Continents, Vol. VIII, IARC Scientific Publications No. 155. Lyon: International Agency for Research on Cancer; 2002.

28. Forman D, Bray F, Brewster DH, et al. Cancer Incidence in Five Continents, Vol. X, IARC Scientific Publication No. 164. Lyon: International Agency for Research on Cancer; 2014.

29. Ahmad OB, Boschi-Pinto C, Lopez AD, et al. Age Standardization of Rates: A New WHO Standard. GPE Discussion Paper Series: No.31. Geneva: World Health Organization, 2001.

30. SAS Institute Inc. SAS/STAT 12.1 User's Guide. Cary, NC: SAS Institute Inc. 7505-12 p.

31. Rosenberg PS, Anderson WF. Age-period-cohort models in cancer surveillance research: ready for prime time? Cancer Epidemiol Biomarkers Prev 2011;20(7):1263-8.

32. Rosenberg PS, Check DP, Anderson WF. A web tool for age-period-cohort analysis of cancer incidence and mortality rates. Cancer Epidemiol Biomarkers Prev 2014;23(11):2296-302.

33. Li QD, Li H, Li FJ, et al. Nutrition deficiency increases the risk of stomach cancer mortality. BMC Cancer 2012;12:315.

34. Abnet CC, Corley DA, Freedman ND, et al. Diet and upper gastrointestinal malignancies. Gastroenterology 2015;148(6):1234-43 e4.

35. Nettle D, Frankenhuis WE, Rickard IJ. The evolution of predictive adaptive responses in human life history. Proc Biol Sci 2013;280(1766):20131343.

36. Zhai $\mathrm{F}$, Wang $\mathrm{H}$, Du S, et al. Lifespan nutrition and changing socio-economic conditions in China. Asia Pac J Clin Nutr 2007;16 Suppl 1:374-82.

37. Zhai F, Wang H, Du S, et al. Prospective study on nutrition transition in China. Nutr Rev 2009;67 Suppl 1:S56-61.

38. Li Y, Hu FB, Ma G. Early life famine exposure and chronic diseases in China. In: Lumey LH, Vaiserman A, editors. Early life nutrition and adult health and development. New York: Nova Science Publishers Inc.; 2013. p. 123-44.

39. Huang $C$, Phillips MR, Zhang $\mathrm{Y}$, et al. Malnutrition in early life and adult mental health: evidence from a natural experiment. Soc Sci Med 2013;97:259-66.

40. Cohen DH, LeRoith D. Obesity, type 2 diabetes, and cancer: the insulin and IGF connection. Endocr Relat Cancer 2012;19(5):F27-45.

41. Lin Y, Ness-Jensen E, Hveem K, et al. Metabolic syndrome and esophageal and gastric cancer. Cancer Causes Control 2015;26(12):1825-34.

42. Bearer CF. The special and unique vulnerability of children to environmental hazards. Neurotoxicology 2000;21(6):925-34.

43. Bearer CF. How are children different from adults? Environ Health Perspect 1995;103 Suppl 6:7-12.

44. Xie SH, Lagergren J. The Male Predominance in Esophageal Adenocarcinoma. Clin Gastroenterol Hepatol 2016;14(3):338-47 e1.

45. Meng X, Qian N, Yared P. The institutional causes of China's Great Famine, 1959-1961. Rev Econ Stud 2015;82:1568-611.

46. Shanghai Statistics Bureau of Statistics. Shanghai Migrant Population Development and Characteristics. 2011 [cited 2016 March 21]; Available from: http://www.statssh.gov.cn/fxbg/201109/232741.html.

47. Shanghai Statistics Bureau of Statistics. Bulletin of 1\% Population Sampling Survey in Shanghai, 2005. 2006 [cited 2016 September 30]; Available from: http://www.stats.gov.cn/tisi/tigb/rkpcgb/dfrkpcgb/200603/t20060320 30365.html. 
Table 1. Crude and age-standardized incidence rates (ASRs) with $95 \%$ confidence intervals (CIs) of gastrointestinal cancers per 100,000 person-years by sites and calendar periods in Shanghai, 1983 - 2007

\begin{tabular}{|c|c|c|c|c|c|c|}
\hline \multirow[t]{2}{*}{ Cancers and calendar periods } & \multicolumn{3}{|c|}{ Males } & \multicolumn{3}{|c|}{ Females } \\
\hline & $\mathbf{N}$ & Crude rate $(95 \% \mathrm{CI})$ & $\operatorname{ASR}(95 \% \mathrm{CI}){ }^{*}$ & $\mathbf{N}$ & Crude rate $(95 \% \mathrm{CI})$ & $\operatorname{ASR}(95 \% \mathrm{CI}){ }^{*}$ \\
\hline \multicolumn{7}{|l|}{ Esophageal cancer } \\
\hline 1983-1987 & 2,868 & $17.1(16.5,17.7)$ & $17.3(16.6,18.1)$ & 1,477 & $9.1(8.7,9.6)$ & $7.4(7.0,7.8)$ \\
\hline 1988-1992 & 2,855 & $15.7(15.1,16.3)$ & $14.3(13.8,14.9)$ & 1,338 & $7.7(7.3,8.1)$ & $5.5(5.2,5.8)$ \\
\hline 1993-1997 & 2,355 & $14.3(13.8,14.9)$ & $11.2(10.7,11.7)$ & 1,189 & $7.5(7.1,8.0)$ & $4.6(4.4,4.9)$ \\
\hline $1998-2002$ & 2,291 & $14.4(13.8,15.0)$ & $11.0(10.5,11.4)$ & 965 & $6.2(5.8,6.6)$ & $3.6(3.4,3.9)$ \\
\hline 2003-2007 & 2,017 & $13.0(12.4,13.5)$ & $7.8(7.5,8.2)$ & 791 & $5.2(4.8,5.5)$ & $2.4(2.3,2.6)$ \\
\hline Net drift $(95 \% \mathrm{CI}), \%$ & \multicolumn{3}{|c|}{$-3.4(-5.1,-1.6)$} & \multicolumn{3}{|c|}{$-6.8(-8.7,-4.8)$} \\
\hline \multicolumn{7}{|l|}{ Gastric cancer } \\
\hline 1983-1987 & 10,213 & $60.8(59.6,62.0)$ & $58.9(57.6,60.1)$ & 4,917 & $30.4(29.6,31.3)$ & $24.8(24.1,25.5)$ \\
\hline 1988-1992 & 10,761 & $59.2(58.1,60.3)$ & $52.1(51.1,53.2)$ & 5,584 & $32.1(31.3,33.0)$ & $23.7(23.1,24.3)$ \\
\hline 1993-1997 & 8,887 & $54.1(53.0,55.3)$ & $41.7(40.8,42.6)$ & 4,756 & $30.1(29.2,30.9)$ & $20.0(19.4,20.6)$ \\
\hline $1998-2002$ & 8,367 & $52.6(51.5,53.7)$ & $39.8(38.9,40.7)$ & 4,839 & $31.2(30.3,32.1)$ & $19.8(19.2,20.4)$ \\
\hline 2003-2007 & 7,959 & $51.2(50.0,52.3)$ & $31.2(30.5,31.9)$ & 4,728 & $30.8(30.0,31.7)$ & $16.3(15.9,16.8)$ \\
\hline Net drift $(95 \% \mathrm{CI}), \%$ & \multicolumn{3}{|c|}{$-3.2(-3.7,-2.7)$} & \multicolumn{3}{|c|}{$-2.2(-2.7,-1.6)$} \\
\hline \multicolumn{7}{|l|}{ Liver Cancer } \\
\hline $1983-1987$ & 6,146 & $36.6(35.7,37.5)$ & $33.8(32.9,34.7)$ & 2,423 & $15.0(14.4,15.6)$ & $12.1(11.6,12.6)$ \\
\hline 1988-1992 & 6,459 & $35.5(34.7,36.4)$ & $30.8(30.0,31.6)$ & 2,579 & $14.8(14.3,15.4)$ & $11.0(10.6,11.5)$ \\
\hline 1993-1997 & 5,844 & $35.6(34.7,36.5)$ & $27.6(26.9,28.3)$ & 2,452 & $15.5(14.9,16.1)$ & $10.1(9.7,10.5)$ \\
\hline $1998-2002$ & 6,001 & $37.7(36.8,38.7)$ & $28.8(28.1,29.6)$ & 2,300 & $14.8(14.2,15.5)$ & $9.6(9.2,10.0)$ \\
\hline 2003-2007 & 6,128 & $39.4(38.4,40.4)$ & $24.3(23.7,25.0)$ & 2,419 & $15.8(15.2,16.4)$ & $8.2(7.9,8.6)$ \\
\hline Net drift $(95 \%$ CI $), \%$ & \multicolumn{3}{|c|}{$-1.8(-2.3,-1.4)$} & \multicolumn{3}{|c|}{$-2.3(-3.0,-1.6)$} \\
\hline \multicolumn{7}{|l|}{ Gallbladder cancer } \\
\hline 1983-1987 & 397 & $2.4(2.1,2.6)$ & $2.3(2.0,2.5)$ & 709 & $4.4(4.1,4.7)$ & $3.5(3.2,3.7)$ \\
\hline 1988-1992 & 577 & $3.2(2.9,3.4)$ & $2.9(2.6,3.1)$ & 971 & $5.6(5.2,6.0)$ & $4.0(3.7,4.3)$ \\
\hline 1993-1997 & 671 & $4.1(3.8,4.4)$ & $3.2(2.9,3.4)$ & 1,142 & $7.2(6.8,7.6)$ & $4.6(4.3,4.9)$ \\
\hline $1998-2002$ & 858 & $5.4(5.0,5.8)$ & $4.2(4.0,4.5)$ & 1,538 & $9.9(9.4,10.4)$ & $6.0(5.7,6.3)$ \\
\hline
\end{tabular}


2003-2007

Net drift (95\% CI), \%

\section{Pancreatic cancer}

\section{3-1987}

1988-1992

1993-1997

1998-2002

2003-2007

Net drift (95\% CI), \%

\section{Colorectal cancer}

1983-1987

1988-1992

1993-1997

1998-2002

2003-2007

Net drift $(95 \%$ CI), \%

$$
5.9(5.5,6.3)
$$

$0.9(-1.9,3.8)$

$3.6(3.4,3.8)$

1,165

1,445

1,534

1,812

2,100

$$
6.9(6.5,7.3)
$$

$8.0(7.5,8.4)$

$9.3(8.9,9.8)$

$11.4(10.9,11.9)$

$13.5(12.9,14.1)$

\section{3,540}

4,926

5,467

6,533

8,137
$21.1(20.4,21.8)$

$27.1(26.4,27.9)$

$33.3(32.4,34.2)$

$41.1(40.1,42.1)$

$52.3(51.2,53.4)$
$0.6(-0.5,1.6)$

$6.8(6.3,7.2)$

$7.1(6.7,7.4)$

$7.2(6.8,7.6)$

$8.7(8.3,9.1)$

$8.3(7.9,8.6)$

$$
0.3(-0.9,1.6)
$$

$20.1(19.4,20.8)$

$24.0(23.3,24.7)$

$25.6(25.0,26.4)$

$31.3(30.5,32.1)$

$32.2(31.5,33.0)$
1,599

$10.4(9.9,11.0)$

$0.4(-1.2,2.0)$

$5.7(5.3,6.1)$

$6.4(6.1,6.8)$

$8.6(8.2,9.1)$

$10.2(9.7,10.8)$

$12.4(11.8,13.0)$

$1.4(0.5,2.4)$

3,473

4,652

5,081

6,339

7,716
$21.5(20.8,22.2)$ $26.8(26.0,27.6)$

$32.1(31.2,33.0)$

$40.9(39.9,41.9)$

$50.3(49.2,51.5)$
$5.1(4.8,5.3)$

$4.6(4.3,4.9)$

$4.6(4.4,4.9)$

$5.4(5.1,5.7)$

$6.2(5.9,6.5)$

$6.3(6.0,6.6)$

$17.5(16.9,18.1)$ $19.9(19.3,20.5)$ $21.5(20.8,22.1)$ $26.2(25.5,26.9)$ $26.7(26.0,27.3)$

* Standardized to the World Health Organization (WHO) World Standard Population 2000. 


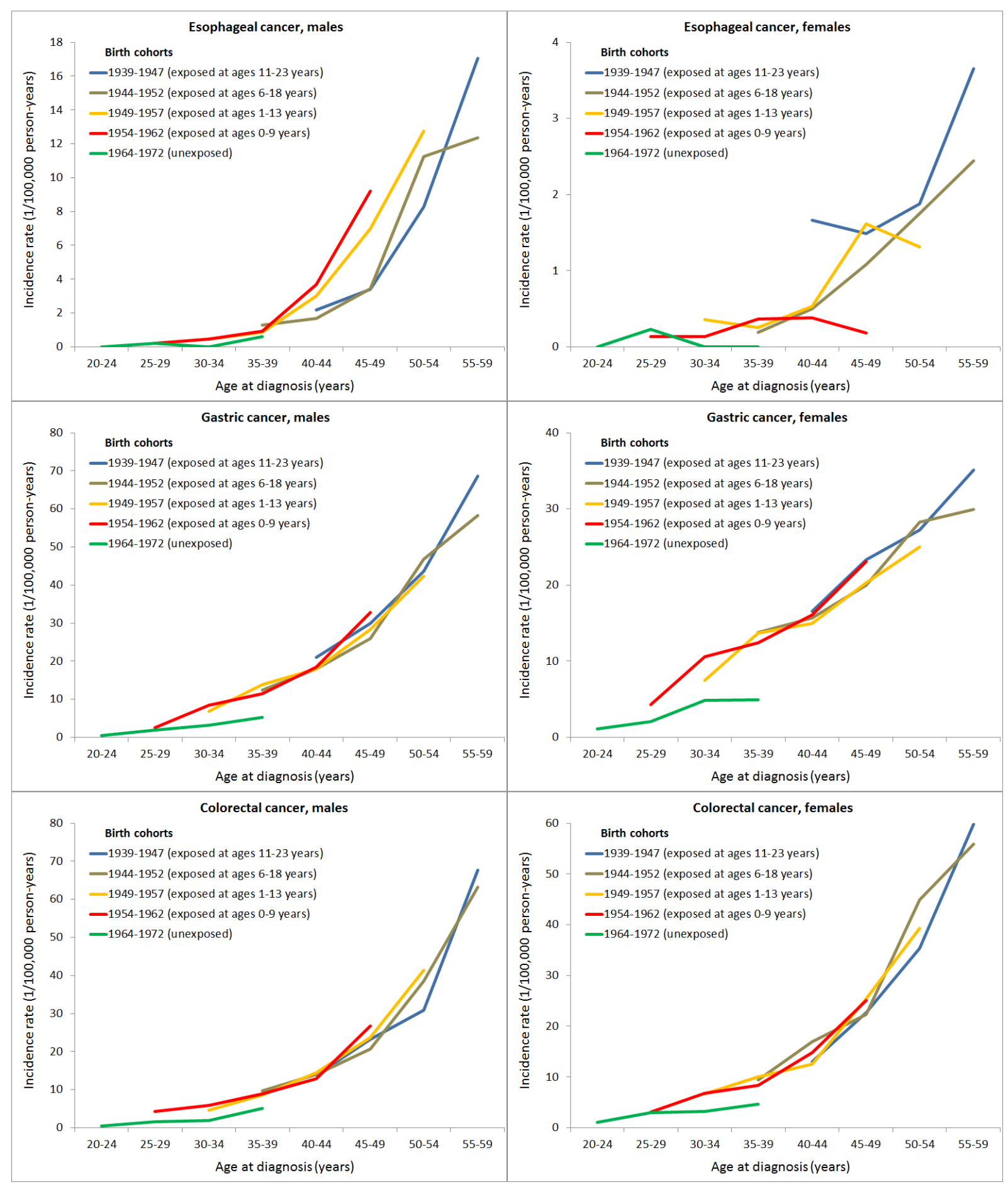

Figure 1. Age-specific incidence rates of esophageal, gastric, and colorectal cancers by sex in birth cohorts exposed to the Chinese famine (1958-1962) in early life and a non-exposed reference birth cohort. 


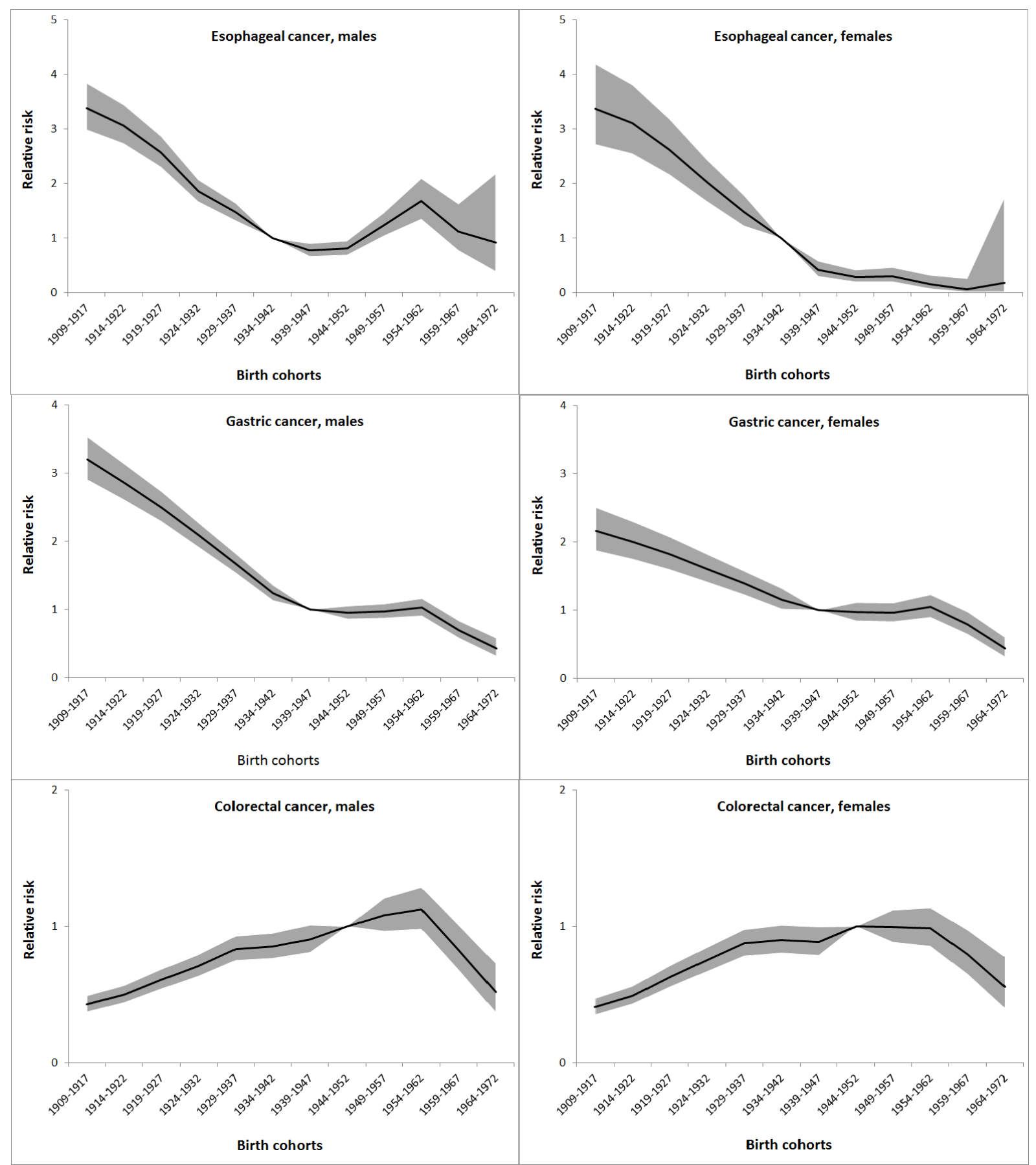

Figure 2. Birth cohort-specific relative risks (black curves) of esophageal, gastric, and colorectal cancers and their $95 \%$ confidence intervals (grey shadowing) by sex obtained from age-period-cohort regression analysis. 

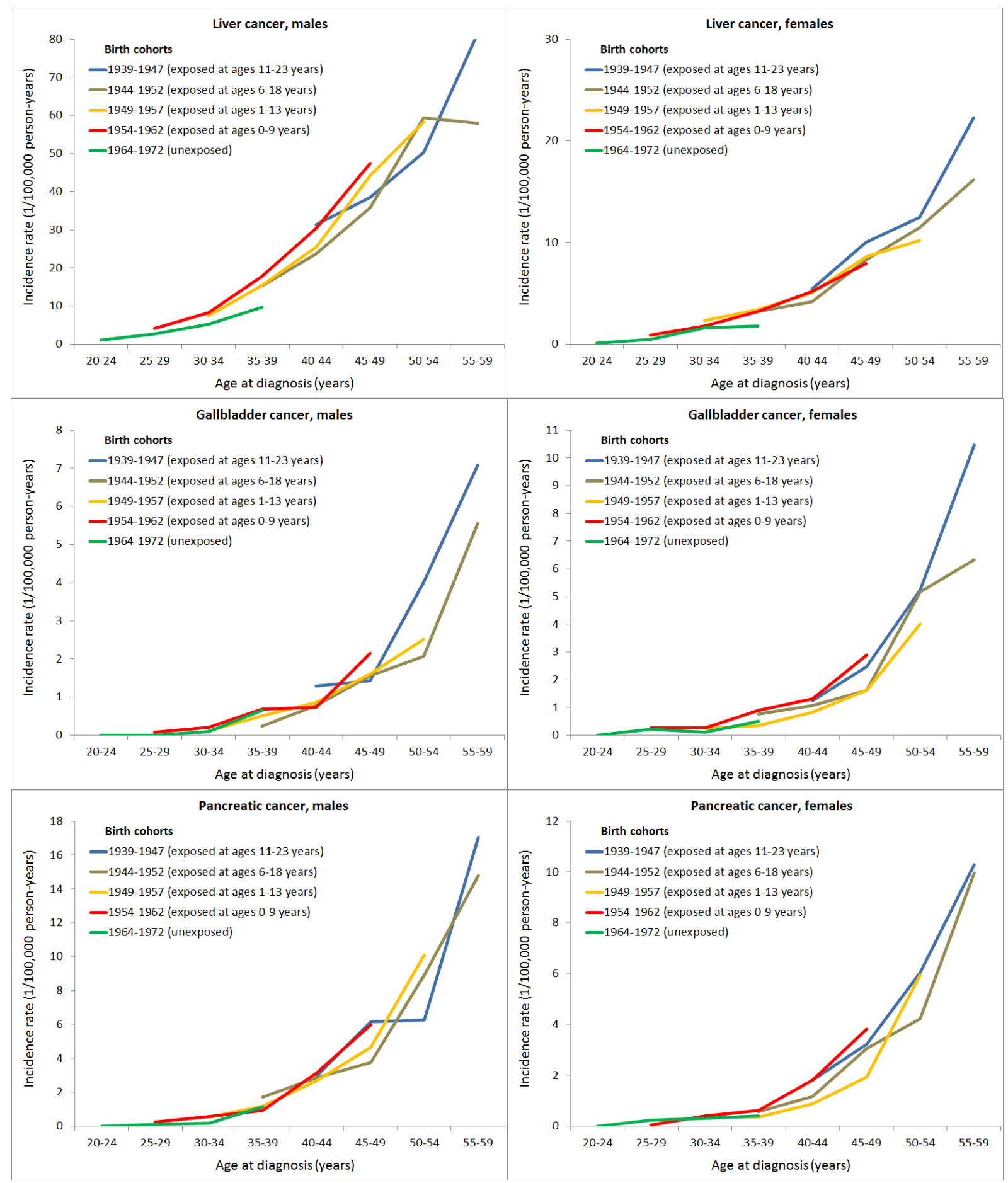

Figure 3. Age-specific incidence rates of liver, gallbladder, and pancreatic cancers by sex in birth cohorts exposed to the Chinese famine (1958-1962) in early life and a non-exposed reference birth cohort. 


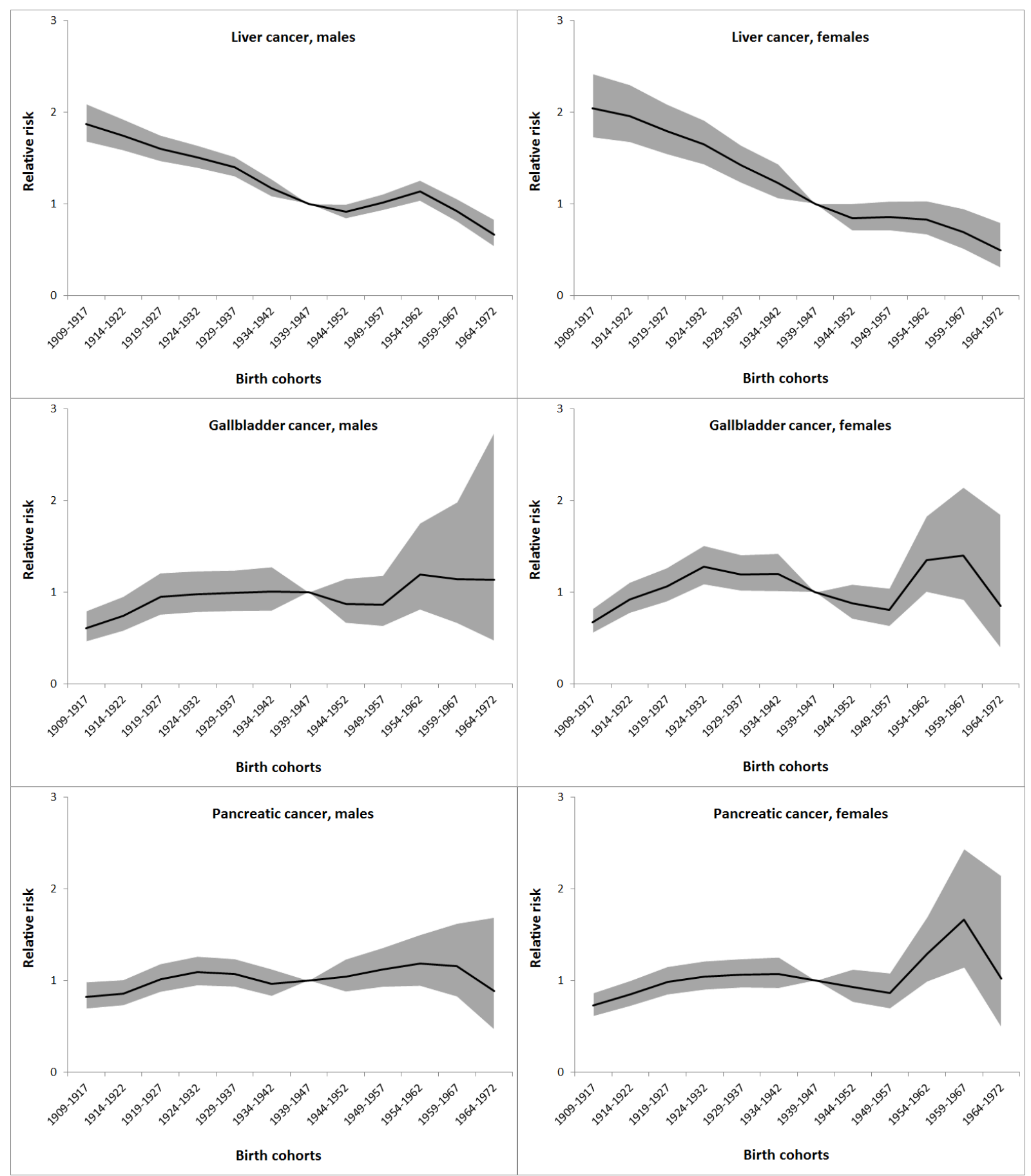

Figure 4. Birth cohort-specific relative risks (black curves) of liver, gallbladder, and pancreatic cancers and their 95\% confidence intervals (grey shadowing) by sex obtained from age-period-cohort regression analysis. 


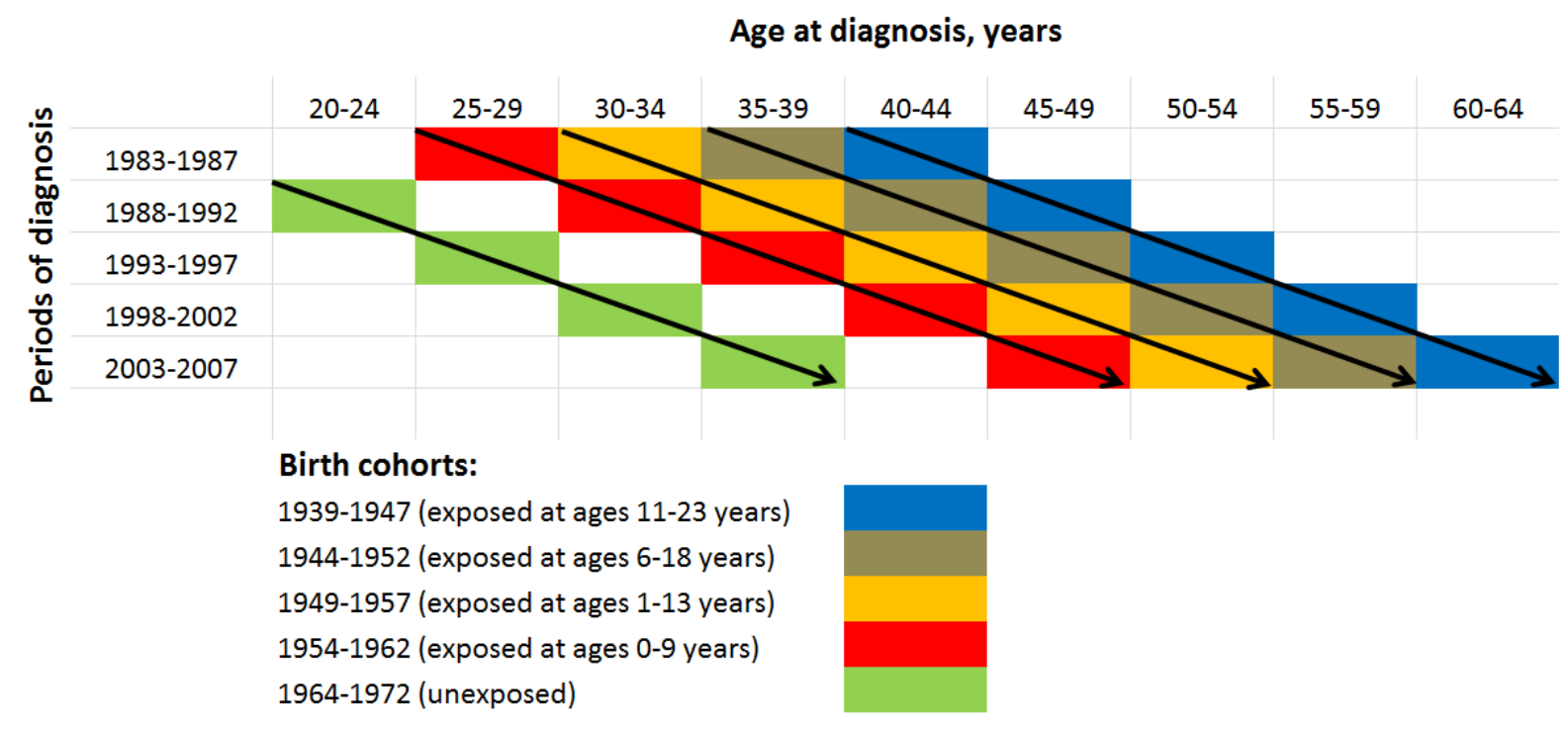

Supplementary Figure 1. Lexis diagram showing the data matrix analyzed with age groups in the columns, calendar time periods in the 